CUBO A Mathematical Journal Vol.15, No 01, (131-149). March 2013

\title{
Planar Pseudo-almost Limit Cycles and Applications to solitary Waves
}

\author{
BOURAMA TONI \\ Virginia State University, \\ Department of Mathematics $\&$ Computer Science, Petersburg VA 23806. \\ btoni@vsu.edu
}

\begin{abstract}
We investigate the existence of pseudo-almost limit cycles, a new class of non-periodicity at the interface of the theories of limit cycles and pseudo-almost periodicity. We determine the conditions of existence for several systems including some pseudo-almost periodic perturbations of the harmonic oscillator and the renowned Liénard systems. We apply to derive the existence of pseudo-almost periodic solitary waves by perturbing first then transforming some hyperbolic and parabolic partial differential equations to Liénard-type equations. Included also are open questions on the co-existence of limit cycles and strictly pseudo-almost periodic limit cycles partitioning the phase space, and the existence of isochronous pseudo-almost limit cycles.
\end{abstract}

\section{RESUMEN}

Investigamos la existencia de ciclos seudo-casi límites, una nueva clase de no-periodicidad en la interfaz de las teorías de ciclos límites y seudo-casi periodicidad. Determinamos condiciones de existencia de muchos sistemas, incluyendo algunas perturbaciones seudocasi periódicas del oscilador armónico y los sistemas de Liénard. Aplicamos las condiciones para derivar la existencia de ondas solitarias seudo-cuasi periódicas, primero perturbando y luego transformando algunas ecuaciones diferenciales parciales hiperbólicas y parabólicas a ecuaciones del tipo Liénard. También se incluyen preguntas abiertas sobre la co-existencia de ciclos límite y estrictamente pseudo-casi periódicos ciclos límite de partición del espacio de fases, y la existencia de isócrono pseudo-casi ciclos límite .

Keywords and Phrases: Limit cycles. Almost and pseudo-almost periodic orbits. Periodic waves. Isochronous systems and Isochrons. Liénard systems. Hyperbolic and parabolic equations.

2010 AMS Mathematics Subject Classification: 34C05, 34C07, 34C27, 34K14 


\section{Introduction}

Limit cycles are used to model the dynamical state of self-sustained oscillations found very often in biology, chemistry, mechanics, electronics, fluid dynamics, etc. See for example [2, 16, 18, 26]. They often arise in many physical systems around a state at which energy generation and dissipation balance. One of the most important limit cycles of our lives is the heartbeat. A spectacular example is the Tacoma Narrows Bridg 1 . and its 1940 dramatic collapse, where the limit cycle drew its energy from the wind and involved torsional oscillations of the roadbed. In Robotics the stable gait to which the repeated dynamic walking pattern converges is modeled as a stable limit cycle, stability easily lost to even small disturbances, evidence of a narrow basin of attracting of the limit cycle.

Planar limit cycles were defined by Poincaré2 in the famous paper Mémoire sur les courbes définies par une équation différentielle [22, using his so-called Method of sections. However much attention in this century has been drawn to the determination of the number, amplitude and configuration of limit cycles in a general nonlinear system, which is still an unsolved problem. This is part of the so-called Hilbert's 16th Problem 3 . A weakened version 4 by Arnold called the tangential Hilbert's problem, concerns the bound on the number of limit cycles which can bifurcate from a first-order perturbation of a Hamiltonian system. [3, 9, 13, 14, 17]

The possibility of a limit cycle on a plane or a two-dimensional manifold is restricted to nonlinear dynamical systems, due to the fact that, for linear systems, $k x(t)$ is also a solution for any constant $k$ if $x(t)$ is a solution. Therefore the phase space will contain an infinite number of closed trajectories encircling the origin, with none of them isolated. Conservative and gradient systems do not have limit cycles, but these systems may exhibit almost or pseudo-almost limit cycles. The most common techniques for predicting the absence or existence of periodicity and limit cycles include the Index Theory, Dulac's Criterion, Poincaré-Bendixson Test, Perturbation and Bifurcation theory, Configuration of limit cycles, the Toroidal Principle. These concepts and related examples could be found in [2, 5, 6, 9, 10, 13, 18, 25]. The nonlinear character of isolated periodic oscillations renders their detection and construction challenging. In mechanical terms the appraisal of the regions of the phase plane where energy loss and energy gain occur might reveal a limit cycle.

Let us emphasize that even though in most studies periodicity has been illustrated more frequently, almost and pseudo-almost periodic oscillations or waves actually occur much more

\footnotetext{
${ }^{1} \mathrm{~A}$ wealth of information including historical and anecdotal facts could be found in http://en.wikipedia.org/wiki/Tacoma-Narrows-Bridge(1940)

2 Jules Henri Poincaré has excelled in all fields of knowledge and is often described as a polymath or The Last Universalist. The famous Poincaré conjecture named after him was finally solved in 2002-2003 by Grigori Perelman who turned down the related prize of $\$ 1,000,000$ !

${ }^{3}$ Determine the maximum number and relative positions of limit cycles in polynomial vector fields of degree $\mathrm{n}$. Stated in 1900, it was only in 1987 that Ecalle and Ilyashenko proved independently the finiteness of that number using the compactification of the phase space to Poincaré disk

${ }^{4}$ The number of limit cycles in a small perturbation of a polynomial Hamiltonian system is given by the number of zeroes of Abelian integrals at least far from polycycles.
} 
frequently than periodic ones. For instance, in the simplest model of harmonic oscillator or mathematical pendulum, as well as for the one-dimensional wave equation, diverse kinds of oscillatory trajectories can be displayed, both periodic and more generally non-periodic.

The theory of almost periodic functions introduced by H. Bohr [6] in the 1920s and extended to pseudo-almost periodicity 5 by Zhang [27] in the 1990s is also connected with problems in differential equations, stability theory, dynamical systems, partial differential equations or equations in Banach spaces. There are several results concerning the existence and uniqueness of almost and pseudoalmost periodic solutions for first-order differential equations, e.g., in [7, 11, 12, 15, 20, 21, 23, 24, 27. But the authors usually derived their results from the existence of bounded solutions.

We extend the theory of limit cycles and pseudo-almost periodicity to that of pseudo-almost limit cycles, isolated pseudo-almost periodic orbits, and we investigate in the current and future work the usual questions of conditions of existence and uniqueness, stability, bifurcation and perturbation, the coexistence of limit cycles and strictly pseudo-almost limit cycles. We also introduce the idea of isochronous pseudo-almost limit cycles and pseudo-almost isochron 6 .

Section 2 overviews the theory of limit cycles recalling the definitions and presenting some classic and concrete examples relevant to our study. In section 3, we develop the concept of pseudoalmost limit cycle, its properties, several illustrative examples including the so-called linear pseudocenter, and existence theorems in the case of the well-known Liénard systems. Section 4 shows the applications of the existence theorems for Liénard systems to obtain pseudo-almost periodic solitary waves for some hyperbolic and parabolic partial differential equations. Finally in section 5 we discuss some directions for future research, and state some open problems, defining in the process the concept of isochronous pseudo-almost limit cycles and pseudo-almost isochrons.

\section{Preliminary Definitions and Examples}

Let the multi-dimensional space $\mathbb{R}^{n}$ represents all the possible states of a system modeling nonlinear phenomena. The dynamics of the system are determined by the values in $\mathbb{R}^{n}$ in terms of the time. That is to say we define an evolution map or flow $\Phi$, smooth on the smooth manifold $\mathbb{R}^{n}$ :

$$
\Phi: \quad \mathbb{R}^{n} \times \mathbf{R} \longrightarrow \mathbb{R}^{n}
$$

such that $\Phi(x, t)=y$ indicates that the state $x \in \mathbb{R}^{n}$ evolved into the state $y \in \mathbb{R}^{n}$ after $t$ units of time, together with the usual flow properties

$$
\Phi(x, 0)=x, \quad \Phi\left(x, t_{1}+t_{2}\right)=\Phi\left(\Phi\left(x, t_{1}\right), t_{2}\right)
$$

\footnotetext{
${ }^{5}$ Any pseudo-almost periodic function is also a Besicovitch almost periodic function

${ }^{6}$ The development of the concept of isochrons and the recognition of their significance is due to Winfree (1980)
} 
The flow $\Phi$ then determines a vector field $\mathcal{X}$ (conversely as well) such that, for $x \in M$

$$
\mathcal{X}(x):=\frac{\partial \Phi}{\partial t}(x, 0)
$$

The orbit or trajectory of the flow through $x \in \mathbb{R}^{n}$ is given by:

$$
\mathrm{O}(\mathrm{x}):=\left\{\Phi_{\chi}(\mathrm{t}):=\Phi(x, \mathrm{t}) \mid \mathrm{t} \in \mathbb{R}\right\}
$$

Definition 2.1. The orbit $\gamma=\mathrm{O}(\mathrm{x})$ based at $\mathrm{x}$ is called a limit cycle if there is a neighborhood $\mathcal{N}$ of $\gamma$ such that $\gamma$ is the only periodic orbit contained in $\mathcal{N}$.

The limit cycle 7 is stable (unstable) if $\omega(s)=\gamma(\alpha(s)=\gamma)$ for any $s \in \mathcal{N}$, that is, $\gamma$ is the $\omega$-limit set $(\alpha-$ limit set) of any point in $\mathcal{N}$. In other words, the limit cycle, isolated periodic orbit of some period $\tau$, is stable (resp. unstable) if it has a neighborhood $\mathcal{N}$ such that, for some distance function $\mathrm{d}$ on $\mathbb{R}^{n}, \mathrm{~d}(\Phi(\mathrm{y}, \mathrm{t}), \gamma) \longrightarrow 0$, as $\mathrm{t} \rightarrow \infty$ (resp. $\left.\mathrm{t} \rightarrow-\infty\right)$, for any $\mathrm{y} \in \mathcal{N}$.

Note that the phase $\varphi=\frac{t}{T_{0}}$ of a limit cycle of period $T_{0}$ refers to the relative position on the orbit, which is measured by the elapsed time (modulo the period) to go from a reference point to the current position on the limit cycle. The most common illustrative examples are from the perturbations of the linear center or linear isochrone.

\subsection{Linear center and its perturbations}

\subsubsection{Poincaré oscillator}

The linear center or linear isochron 8

$$
\dot{x}=-y, \quad \dot{y}=x,
$$

where the origin of the plane is surrounded by a continuum of periodic orbits (not isolated) given by $x^{2}+y^{2}=c>0$, is perturbed into the following system, in polar coordinates $(r, \theta)$

$$
\dot{r}=r(1-r), \quad \dot{\theta}=1
$$

The circle $r=1$ is a $2 \pi$-periodic orbit and is unique. It is therefore a limit cycle. Moreover $r$ is a monotone function on each orbit ( $\dot{r}>0$ inside and $<0$ outside) so that all non constant orbits tend towards the limit cycle which is therefore stable $[9,2,18,10$

\footnotetext{
${ }^{7}$ A limit cycle actually controls the behavior of neighboring orbits, attracting/repelling on both sides, or attracting on one side and repelling on the other

${ }^{8}$ The term isochrone refers to the fact that all the periodic orbits in the continuum have the same constant period normalized to $2 \pi$.

${ }^{9}$ The Poincaré's oscillator has been considered a model of biological oscillations, in particular with respect to the effects of periodic stimulation of cardiac oscillators

${ }^{10}$ The isochrons here are radial lines from which the trajectories evolve to equal phase
} 


\subsubsection{Limit cycles Annulus}

The linear center could also be perturbed into a system to generate several limit cycles as in the following example. The $\mathrm{C}^{\infty}$-system

$$
\dot{x}=-y+x p(x, y), \quad \dot{y}=x+y p(x, y),
$$

where

$$
p(x, y)=\sin \left(\frac{1}{x^{2}+y^{2}}\right) e^{-\frac{1}{x^{2}+y^{2}}},
$$

has an infinite number of limit cycles

$$
\gamma_{n}: \quad x^{2}+y^{2}=\frac{1}{n \pi}, \quad n \in \mathbb{N}
$$

accumulating at the origin. [9]

\subsubsection{Remarks}

The linear center is a continuum of periodic orbits encircling a critical point. The perturbation in examples 1 and 2 has in fact destroyed these orbits to give birth to respectively a unique limit cycle in example 1 , and an accumulating family of limit cycles in example 2 . We will see below that a time-dependent pseudo-almost perturbation could lead to the emergence of the so-called pseudo-almost limit cycles.

\section{Pseudo-almost limit cycles}

\subsection{Introductory Concepts}

Let $\mathcal{C}\left(\mathbb{R} \times \Omega, \mathbb{R}^{n}\right), \quad \Omega \subset \mathbb{R}^{n}$ open, be the Banach space of bounded continuous functions $\phi(t, x)$ endowed with the norm $\|\phi\|=\sup _{t \in \mathbb{R}, x \in \Omega}|\phi(t, x)|$. The set $\mathcal{C}\left(\mathbb{R} \times \Omega, \mathbb{R}^{n}\right)$ is a subset of the more general space $\mathcal{L}_{\mathrm{b}}\left(\mathbb{R} \times \Omega, \mathbb{R}^{\mathfrak{n}}\right)$ of all Lebesgue measurable and bounded functions.

Definition 3.1. A function $\mathrm{f}$ in $\mathcal{L}_{\mathrm{b}}\left(\mathbb{R} \times \Omega, \mathbb{R}^{\mathrm{n}}\right)$ is said to be ergodic if for every compact subset $\mathrm{K} \subset \Omega$ the mean defined by

$$
\mathcal{M}(f):=\lim _{T \rightarrow \infty} \frac{1}{2 T} \int_{-T}^{T} f(t, x) d t,
$$

exists uniformly for $\mathrm{x} \in \mathrm{K}$.

We say that the function has a vanishing mean if $\mathcal{M}(\mathrm{f})=0$. Let $\mathcal{E}\left(\mathbb{R} \times \Omega, \mathbb{R}^{\mathfrak{n}}\right)$ denote the space of all ergodic functions on $\mathbb{R} \times \Omega$. Note in passing that not all uniformly continuous bounded 
functions on $\mathbb{R}$ are ergodic. For instance the function

$$
f(t)=\left\{1-t^{2}, \quad \text { for }|t|<1, \quad \text { and } \sin \left(\log \left(\frac{1}{t^{2}}\right)\right), \text { for }|t| \geq 1,\right\}
$$

is uniformly continuous in $\mathbb{R}$, but not ergodic.

In the space $\mathcal{L}\left(\mathbb{R} \times \Omega, \mathbb{R}^{n}\right)$ of all Lebesgue measurable functions on $\mathbb{R} \times \Omega$, we consider next the following subspace $\mathcal{L}_{0}$ of all functions $\phi: \mathbb{R} \times \Omega \rightarrow \mathbb{R}^{n}$ such that $\forall x \in \Omega, \tilde{\phi}():.=\phi(., x)$ is Lebesgue measurable on $\mathbb{R}$ with $\mathcal{M}(|\tilde{\phi}|)=0$, and $\mathcal{M}(|\phi|)=0$.

For example the function

$$
\phi(t)=t|\sin \pi t|^{t^{N}}, \quad N>6,
$$

is unbounded, Lebesgue measurable with vanishing mean $\mathcal{M}$.

The unbounded and discontinuous function

$$
\phi(t):=\{\sqrt{n}, \quad n \leq t \leq n+1 / n, \quad \text { and } 0, \quad \text { otherwise }\}
$$

is also an element of $\mathcal{L}_{0}$. Indeed we have $\lim _{T \rightarrow \infty} \frac{1}{2 T} \int_{-T}^{T}|\phi(t)| d t=\lim _{n \rightarrow \infty} \frac{1}{n} \sum_{k=1}^{n} \frac{1}{\sqrt{k}}=0$.

Definition 3.2. The orbit $\mathrm{O}\left(\mathrm{x}_{0}\right)$ based at $\mathrm{x}_{0}$ as defined above is called a pseudo-almost limit cycle if it is isolated, and more importantly if the function $\Phi():.=\Phi_{x_{0}}():. \quad \mathbb{R} \longrightarrow \mathbf{R}^{n}$ defining the orbit is pseudo-almost periodic in the following sense: $\forall \epsilon>0, \exists \delta=\delta(\epsilon)>0$, a relatively dense subset $\mathcal{D}_{\epsilon} \subset \mathbb{R}$, a subset $\mathrm{C}_{\epsilon} \subset \mathbb{R}$, such that:

(1) For $\mathrm{m}$ the Lebesgue measure on $\mathbb{R}$,

$$
\lim _{t \rightarrow \infty} \frac{m\left(C_{\epsilon} \cap[-t, t]\right)}{2 t}=0, \quad\left(C_{\epsilon} \text { is called an ergodic zero set }\right)
$$

(2) Let $\mathrm{T}_{\tau} \Phi$ denotes the translate of $\Phi$ by $\tau$, that is, $\left(\mathrm{T}_{\tau} \Phi(\mathrm{t})\right):=\Phi(\mathrm{t}+\tau)$. Then

$$
\left\|\left(\mathrm{T}_{\tau} \Phi\right)(\mathrm{t})-\Phi(\mathrm{t})\right\|<\epsilon, \quad \tau \in \mathcal{D}_{\epsilon}, \quad \mathrm{t}, \mathrm{t}+\tau \in \mathbf{R}-\mathrm{C}_{\epsilon},
$$

(3) Finally

$$
\left|t_{1}-t_{2}\right|<\delta \Longrightarrow\left\|\Phi\left(t_{1}\right)-\Phi\left(t_{2}\right)\right\|<\epsilon, \quad t_{1}, t_{2} \in \mathbb{R}-C_{\epsilon} .
$$

Denote $\mathcal{P} \mathcal{A}$ the space of pseudo-almost periodic functions. These functions satisfy the following properties widely available in the relevant literature. [11, 12, 27.

\subsubsection{Some properties of pseudo-almost periodicity}

We first give an equivalent definition of a pseudo-almost periodic function, in particular in the space $\mathcal{C}\left(\mathbb{R} \times \Omega, \mathbb{R}^{n}\right)$, with the restriction of $\mathcal{L}_{0}$ to the space $\mathcal{E}_{0}$ containing all functions $\phi \in \mathcal{C}\left(\mathbb{R} \times \Omega, \mathbb{R}^{n}\right)$ 
such that

$$
\lim _{T \rightarrow \infty} \frac{1}{2 T} \int_{-T}^{T}|\phi(t, x)| d t=0,
$$

uniformly in $x \in \Omega$.

Definition 3.3. A function $\mathrm{f}: \mathbb{R} \times \Omega \longrightarrow \mathbb{R}^{n}$ is called pseudo-almost periodic in $\mathrm{t}$ uniformly on compact subsets $\mathrm{K}$ of $\Omega$ if it has a unique decomposition in the form

$$
f(t, x)=a(t, x)+e(t, x),
$$

where the component $\mathrm{a}$ is almost periodic, and the component $\mathrm{e} \in \mathcal{E} \subset \mathcal{L}_{0}$ is called the ergodic perturbation of $\mathrm{f}$. Recall that $\mathrm{a}$ is almost periodic if it satisfies the so-called Bohr's property. That is: $\forall \epsilon>0 \exists l=l(\epsilon)$ such that any interval $(\mathrm{t}, \mathrm{t}+\mathrm{l}) \subset \mathbb{R}$ contains a number $\tau_{\epsilon}$, the $\epsilon$-almost period or $\epsilon-$ translation number, such that:

$$
\|f(t+\tau, x)-f(t, x)\|<\epsilon, \quad t \in \mathbb{R}, x \in \Omega .
$$

We have the following properties relevant to our study and details could be found in Zhang [27] and also in [11, 12].

(1) For $f \in \mathcal{P} \mathcal{A}$, the range $f(\mathbb{R}, K):=\{f(t, x) \mid t \in \mathbb{R}, x \in K\}$ is bounded for every bounded subset $\mathrm{K} \subset \Omega$.

(2) The function $f(t,.) \in \mathcal{P} \mathcal{A}$ is uniformly continuous in each bounded subset of $\Omega$ uniformly in t.

(3) When the ergodic zero set $C_{\epsilon}=\emptyset$, the space $\mathcal{P} \mathcal{A}$ coincides with the space $\mathcal{A P}$ of almost periodic functions.

(4) If both functions $f$ and its derivative $f^{\prime}$ are pseudo-almost periodic, with $f=a+e$ and $f^{\prime}=a^{\prime}+e^{\prime}$, where $a$ and $a^{\prime}$ in $\mathcal{P A}$ and $e$ and $e^{\prime}$ in $\mathcal{L}_{0}$, then the functions $a$ and $e$ are differentiable with $a^{\prime}=a$ and $e^{\prime}=e$.

(5) The space $\mathcal{P} \mathcal{A}$ is convolution invariant with the space $\mathrm{L}^{1}(\mathbb{R})$ of integrable functions on $\mathbb{R}$.

\subsubsection{Illustrative Examples}

We present some by now classic examples of pseudo-almost periodic functions. See also [12, 27]. We include here their graphics.

(1) Example 1 The function

$$
\phi_{1}(t)=\sin t+\sin \sqrt{2} t+\frac{e^{-|t|}}{1+t^{2}}
$$


has the almost periodic component $a(t)=\sin t+$ $\sin \sqrt{2} t$, and the ergodic perturbation $e(t)=$ $\frac{e^{-|t|}}{1+t^{2}}$. We represent it along with its components in Figure 1.

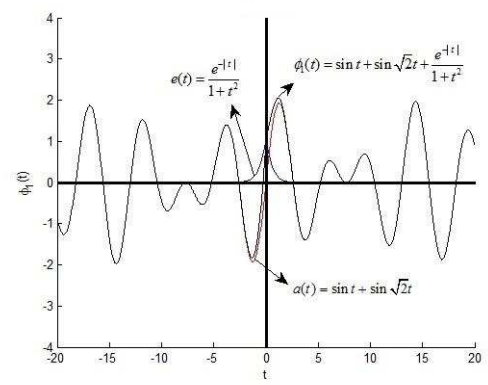

Figure 1: $\phi_{1}(t)=\sin t+\sin \sqrt{2} t+\frac{e^{-|t|}}{1+t^{2}}$

(2) Example 2 We have also the function

$$
\phi_{\omega}(t)=I_{1}(t)+I_{2}(t), \quad \omega \neq 0
$$

with the almost periodic component

$$
I_{1}(t)=\int_{-\infty}^{\infty} h(t-s)(\sin s+\sin \sqrt{2} s) d s, \quad h \in L^{1}(\mathbb{R})
$$

and the ergodic component

$$
I_{2}(t)=\int_{-\infty}^{\infty} \frac{h(t-s)}{s^{2}+\omega^{2}} d s
$$

We take $h(t)=t^{2}$, in $L^{1}(\mathbb{R}), \omega=1$ to illustrate in Figure 2 .
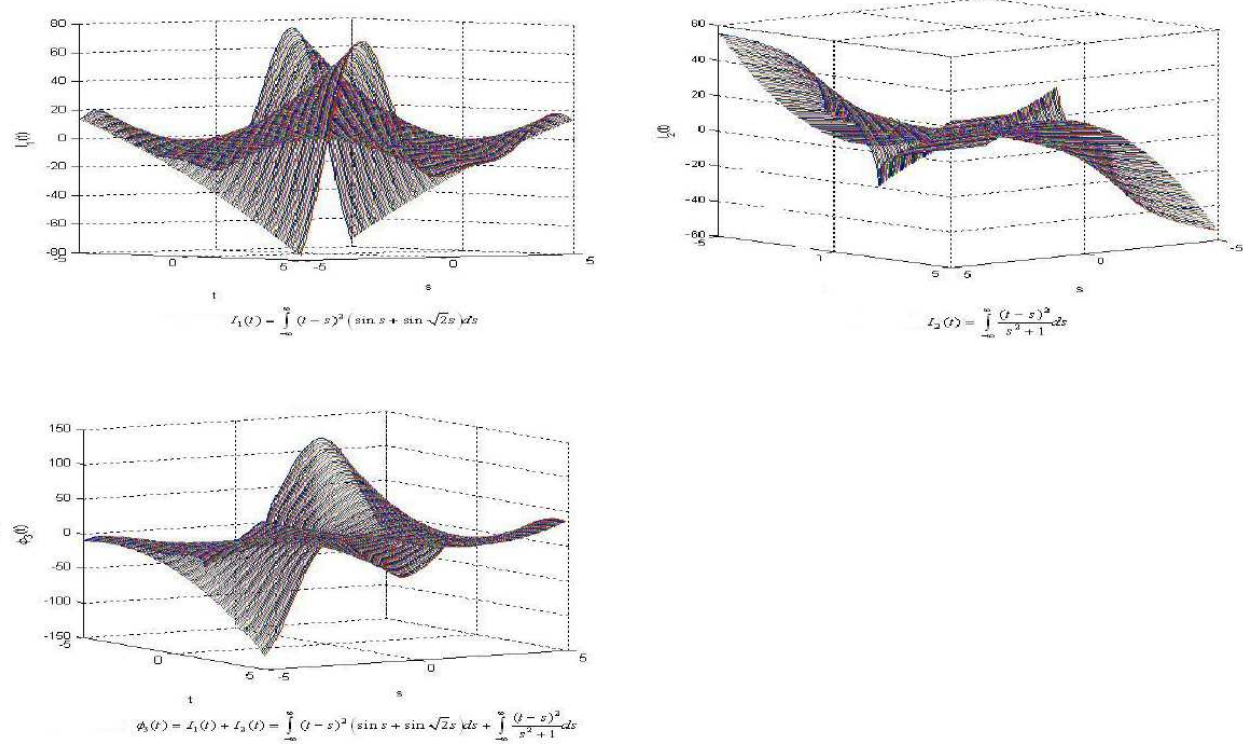

Figure 2: $\phi_{\omega}(\mathrm{t})=\mathrm{I}_{1}(\mathrm{t})+\mathrm{I}_{2}(\mathrm{t})$ 


\subsection{Existence of Pseudo-almost limit cycles}

First note that a periodic or almost periodic function is also pseudo-almost periodic with a zero ergodic perturbation. Consequently a limit cycle is also an almost or a pseudo-almost limit cycle, but not inversely To make the distinction, we will call strictly pseudo-almost limit cycles those pseudo-almost limit cycles that are not limit cycles.

We start with the case of the linear pseudo-almost center.

\subsubsection{Linear pseudo-almost center: an example}

Let $\mathrm{p}(\mathrm{t}) \in \mathcal{P} \mathcal{A}(\mathbb{R}, \mathbb{C})$ be a complex-valued pseudo-almost periodic function defined on the real numbers, and consider the differential equation (see also [1])

$$
\dot{x}(t)=-\alpha x(t)+p(t), \quad \alpha>0 .
$$

Define a kernel

$$
K(t)=\left\{\begin{array}{l}
0, \quad \text { for } t<0 \\
e^{-\alpha t}, \quad \text { for } t \geq 0
\end{array}\right\}
$$

We have $\mathrm{K} \in \mathrm{L}^{1}(\mathbb{R}, \mathbb{C})$. Thus the convolution

$$
x_{\alpha}(t)=(K * p)(t)=e^{-\alpha t} \int_{-\infty}^{t} e^{\alpha s} p(s) d s
$$

is also in $\mathcal{P} \mathcal{A}(\mathbb{R}, \mathbb{C})$, for every $\alpha>0$. Indeed the space $\mathcal{P} \mathcal{A}$ is convolution invariant with $\mathrm{L}^{1}$. The equation being linear, it results in the existence of a continuum of parameterized pseudo-almost periodic solutions which we called linear pseudo-almost center. Therefore these solutions are not isolated, and are not pseudo-almost limit cycles.

A graphical representation for the case $K(t)=t^{2}, \quad p(t)=\sin t+\sin \sqrt{2} t, \quad \alpha=1,2,3,4$ is given in Figure 3.

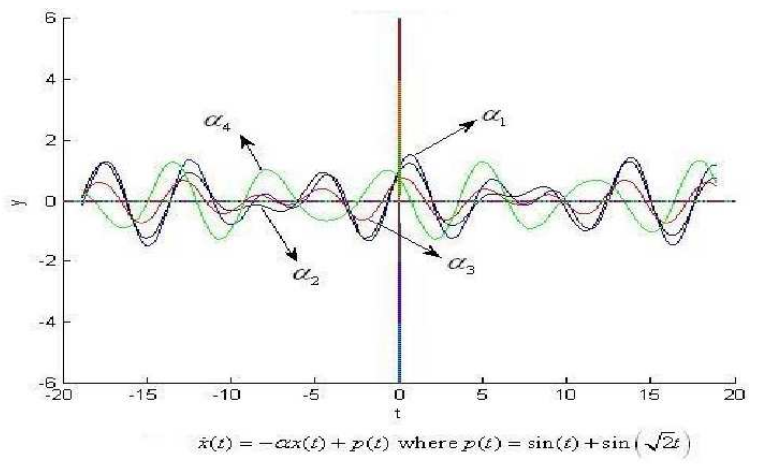

Figure 3: $x_{\alpha}(t)=(K * p)(t)=e^{-\alpha t} \int_{-\infty}^{t} e^{\alpha s} p(s) d s . K(t)=t^{2}, p(t)=\sin t+\sin \sqrt{2} t$ 


\subsubsection{Pseudo-almost periodic perturbations of the harmonic oscillator}

Consider the forced oscillations of the harmonic oscillator given by

$$
\ddot{x}(t)+x(t)=f(t)
$$

where the forcing term is

$$
f(t)=-\sin \sqrt{2} t+\frac{t^{2}\left(t^{2}+4\right)}{\left(t^{2}+1\right)^{3}}
$$

or equivalently, for $\dot{x}=y$

$$
\dot{x}=y, \quad \dot{y}=-x+f(t)
$$

Clearly the function explicitly given by

$$
x(t)=\sin t+\sin \sqrt{2} t+\frac{1}{t^{2}+1}
$$

is the unique solution of the equation and it is one of the classic examples of pseudo-almost periodic function that is not periodic. See also [1]. Therefore we obtain an explicit example of pseudoalmost limit cycle.

Figure 4 gives the phase portrait of $(3.19 a)$ and the graph of the pseudo-almost periodic function in (3.20).
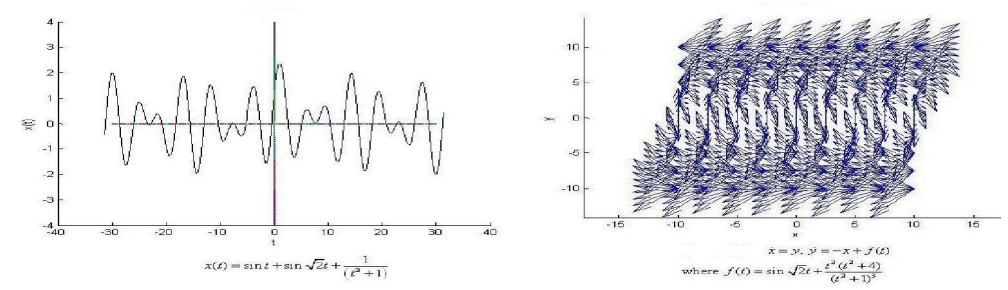

Figure $4 \ddot{x}(t)+x(t)=-\sin \sqrt{2} t+\frac{t^{2}\left(t^{2}+4\right)}{\left(t^{2}+1\right)^{3}}$

We further illustrate the theory of pseudo-almost limit cycles with the well-known Liénard systems.

\subsection{Liénard pseudo-almost limit cycles}

Liénard equation, which also generalizes the famous Van der Pol oscillator, is ubiquitous in the study of nonlinear systems. Consider the one-parameter family of forced Liénard systems

$$
\ddot{x}+f(x) \dot{x}+g(x)=\mu h(t)
$$


or equivalently

$$
\begin{aligned}
& \dot{x}=y-F(x), \\
& \dot{y}=-g(x)+\mu h(t),
\end{aligned}
$$

where $f, g$, are two functions generally nonlinear, continuous and differentiable from $\mathbb{R}$ to $\mathbb{R}$, and $h$ is a time-dependent continuous functions on $\mathbb{R}, \mu \geq 0$ a small real parameter, and $F(x):=\int_{0}^{x} f(s) d s$.

For the homogeneous Liénard systems at $\mu=0$ we recall the following classical result. See more details in, e.g., 5, 10, 18,

Theorem 3.4. If the homogeneous Liénard systems satisfy the following conditions:

(1) $\mathrm{f}(\mathrm{x})$ is continuous, even and $\mathrm{f}(0)<0$.

(2) $\mathrm{g}(\mathrm{x})$ is locally Lipschitz, odd, and such that $\mathrm{xg}(\mathrm{x})>0$ for $\mathrm{x} \neq 0$.

(3) $\mathrm{f}(\mathrm{x})$ has a unique positive zero at $\mathrm{x}=\mathrm{b}$, and it increases at $\infty$ for $\mathrm{x}>\mathrm{b}$.

Then there exists a unique stable limit cycle.

Therefore this theorem provides conditions under which there exists, for the unperturbed Liénard systems, a unique limit cycle, isolated periodic orbit controlling the behavior of neighboring trajectories. We next show that we could subject some classes of Liénard systems to perturbations that, in fact, destroy the limit cycles to give birth to strictly pseudo-almost limit cycles under suitable conditions.

We study system (3.21) or its equivalent form (3.22) under the following additional assumptions:

$\mathrm{L}_{1}: \mathrm{f}(\mathrm{x})>0$, in $\mathbb{R}$, with $\mathrm{F}(\mathrm{x}) \operatorname{sgn} x \rightarrow \infty$ as $|x| \rightarrow \infty$.

$\mathrm{L}_{2}: x \mathrm{~g}(x)>0$ for $x \neq 0, \mathrm{G}(x) \rightarrow \infty$ as $|x| \rightarrow \infty$, with $\mathrm{G}(x):=\int_{0}^{x} \mathrm{~g}(\mathrm{~s}) \mathrm{ds}$.

$\mathrm{L}_{3}:|\mathrm{h}(\mathrm{t})| \leq \mathrm{K}$, and $|\mathrm{H}(\mathrm{t})| \leq \mathrm{K}$, with $\mathrm{H}(\mathrm{t})=\int_{0}^{\mathrm{t}} \mathrm{h}(\mathrm{s}) \mathrm{ds}, \mathrm{t} \in \mathbb{R}$, and $\mathrm{K}$ a positive constant.

$\mathrm{L}_{4}: \mathrm{g}^{\prime}(\mathrm{x})>0$, and $\mathrm{g}^{\prime \prime}(\mathrm{x})$ exists and is bounded.

It is known that, under such assumptions, for $0<\mu \ll 1$, there exists in the xy-plane a region $\mathrm{R}$ bounded by a regular simple curve $\left(\mathrm{C}^{1}\right.$ except possibly at a finite number of points) such that:

(1) For every solution $\gamma(t)=(x(t), y(t))$ of system (3.21) there is a value $t_{0}$ such that $\gamma\left(t_{0}\right) \in R$.

(2) If, for a value $t_{0}$ of $t$, we have $\gamma\left(t_{0}\right) \in R$, then we have also $\gamma(t) \in R$, for $t \geq t_{0}$. That is, solutions entering the set cannot leave it for increasing time.

Moreover the region $R$ depends only on the functions $f(x), g(x), h(t)$, the parameter $\mu$ and the constant K. Equivalently, the region R may be described by the inequalities $|x(t)| \leq x_{0} \quad|\dot{x}(t)| \leq v_{0}$, for a solution $x(t)$ of the equation (3.22), and where $x_{0}$ and $v_{0}$ are constants independent of $\mu$. See, 
for example, 7, 15, 21, 23. In other words, under the above conditions the solutions ultimately settle in a $C^{1}$-bounded region $\mathrm{R}$ in $\mathbb{R}^{2}$. Actually we obtain

Lemma 3.5. Assume the conditions $\mathrm{L}_{1}, \ldots, \mathrm{L}_{4}$. Let $\gamma(\mathrm{t})=(\mathrm{x}(\mathrm{t}), \mathrm{y}(\mathrm{t}))$ be a solution of the system, and $\tilde{\gamma}(\mathrm{t})=(\tilde{\mathrm{x}}(\mathrm{t}), \tilde{\mathrm{y}}(\mathrm{t}))$ either another solution of the system or a solution of an associated system with a sufficiently small perturbation $\overline{\mathrm{h}}(\mathrm{t})$ of the forcing term $\mathrm{h}(\mathrm{t})$. Then we have

$$
\lim _{t \rightarrow \infty}|\tilde{\gamma}(t)-\gamma(t)|=0,
$$

Moreover there exists a unique solution $\mathrm{x}(\mathrm{t})$ for all $\mathrm{t} \in \mathbb{R}$.

Proof. Let $\gamma(t)=(x(t), y(t))$ a solution of the system, and $\tilde{\gamma}(t)=(\tilde{x}(t), \tilde{y}(t))$ either another solution of the system or a solution of an associated system with a sufficiently small perturbation $\bar{h}(t)$ of the forcing term $h(t)$.

$$
\lim _{t \rightarrow \infty}|\tilde{\gamma}(t)-\gamma(t)|=0,
$$

is equivalent to

$$
\lim _{t \rightarrow \infty}|\tilde{x}(t)-x(t)|=0=\lim _{t \rightarrow \infty}|\tilde{y}(t)-y(t)| .
$$

Upon the change of variables $u(t)=\tilde{x}(t)-x(t), \quad v(t)=\tilde{x}(t)-y(t)$, we obtain the system

$$
\begin{gathered}
\dot{u}(t)=v(t)-\varphi(t) u(t) \\
\dot{v}(t)=-\psi(t) u(t)+\mu \Delta h(t),
\end{gathered}
$$

where

$$
\varphi(t)=\frac{F\left(x_{2}\right)-F\left(x_{1}\right)}{x_{2}-x_{1}}, \quad \psi(t)=\frac{g\left(x_{2}\right)-g\left(x_{1}\right)}{x_{2}-x_{1}} .
$$

Note that the function $f, g^{\prime}$ and $g^{\prime \prime}$ are bounded on the compact region R. For sufficiently small values of the parameter $\mu \ll 1$, we can construct a Lyapunov-type quadratic form

$$
V(t, u, v)=\psi(t) u^{2}+v^{2}-2 c u v,
$$

with $c>0$ chosen small enough for $V(t, u, v)$ to be positive definite such that

$$
V(t, u, v) \geq c\left(u^{2}+v^{2}\right)
$$

c a positive constant, and such that

$$
\dot{V}(t, u, v)+c V(t, u, v)<0 .
$$

Actually we have

$$
\frac{d V}{d t}(t, u, v)=-2(\varphi \psi-\dot{\psi}-2 c \psi) u^{2}-2 c v^{2}+2 c \varphi u v,
$$

yielding

$$
\tilde{V}(t, u, v):=\dot{V}(t, u, v)+c V(t, u, v)=-(2 \varphi \psi-\dot{\psi}-3 c \psi) u^{2}+2 c(\varphi-c) u v-c v^{2} .
$$


The quadratic form $\tilde{V}(t, u, v)$ can be made negative definite by taking the constant $\mathrm{c}$ such that

$$
c<\frac{2 \varphi \psi-\dot{\psi}}{3 \psi}, \quad c\left(3 \psi+(\varphi-c)^{2}\right)<2 \varphi \psi-\dot{\psi},
$$

which entails

$$
\dot{V}(t, u, v)<V\left(t_{0}\right) e^{-c\left(t-t_{0}\right)} .
$$

Therefore $\mathrm{V}(\mathrm{t}) \rightarrow 0$ as $\mathrm{t} \rightarrow \infty$, implying that $\mathrm{u} \rightarrow 0$ and $v \rightarrow 0$. The constant $\mathrm{c}$ is appropriately chosen so that, when $|\Delta h(t)|=|\tilde{h}(t)-h(t)| \rightarrow 0$, we can make $V(t) \rightarrow 0$ for $t \rightarrow \infty$. That is, the solutions of the system of the perturbed forcing term ultimately converge to the solutions of the original system.

Next let $\gamma(t)=(x(t), y(t))$ be one of these solutions which settled in $R$ for $t \geq t_{0}$. We then define the sequence of solutions $\gamma_{n}(t)=\gamma(t+n)=\left(x_{n}(t), y_{n}(t)\right), \quad t \geq t_{0}-n$. The sequence is therefore equicontinuous and uniformly bounded. Consequently we can extract a subsequence $\gamma_{n_{k}}(t)$ converging uniformly to a solution $\bar{\gamma}(t)=(\bar{x}(t), \bar{y}(t))$ lying completely in $R$ for all $t \in \mathbb{R}$. $\left(\lim _{n \rightarrow \infty}\left(t_{0}+n, \infty\right)=(-\infty, \infty)\right)$. And of course $\bar{\gamma}(t)$ is unique.

\subsubsection{Remarks}

Indeed the proof of the theorem actually accomplishes the followings: the solutions of the system associated to the perturbed forcing term ultimately converge to the solutions of the original system; moreover, under the assumptions above, only one solution of the system settles in the bounded region $\mathrm{R}$ for all time in $\mathbb{R}$; ; as we show below, that single solution will be of the same nature as the forcing term, when it becomes pseudo-almost periodic.

In a previous work, 24 the case of the pseudo-almost periodic forcing was presented as a corollary to that of almost periodic forcing; here we present a more elegant and self-contained proof drawing from the above definitions of pseudo-almost periodicity, definitions not used in the cited work.

We state and prove

Theorem 3.6. Assume the forcing term $\mathrm{h}(\mathrm{t})$ is a pseudo-almost periodic function. Then under the conditions $\mathrm{L}_{1}, \ldots, \mathrm{L}_{4}$, the forced Liénard system exhibits a unique asymptotically stable pseudoalmost limit cycle.

Proof. The proof is based on the previous lemma, including the existence of a unique solution enclosed in $\mathrm{R}$ for all time. First assuming the forcing term $h(t)$ is pseudo-almost periodic entails from the definition above that, for any arbitrary $\epsilon$, there exists $\delta=\delta(\epsilon)$, an $\epsilon$-pseudo-almost period $\tau \in \mathcal{D}_{\epsilon}$, a relatively dense set in $\mathbb{R}$ such that

$$
\|h(t+\tau)-h(t)\|<\epsilon, \quad t, t+\tau \in \mathbb{R}-C_{\epsilon}
$$


and

$$
\left|t_{1}-t_{2}\right|<\delta \Longrightarrow\left\|h\left(t_{1}\right)-h\left(t_{2}\right)\right\|<\epsilon, \quad t_{1}, t_{2} \in \mathbb{R}-C_{\epsilon},
$$

where $C_{\epsilon}$ is the ergodic zero set defined above. For such an $\epsilon-$ pseudo-almost period consider the unique solution $\bar{\gamma}(\mathrm{t})$ given in the previous lemma that settles in $\mathrm{R}$ for all time $\mathrm{t} \in(-\infty, \infty)$, and the associated function $\bar{\gamma}(t+\tau)=(\bar{x}(t+\tau), \bar{y}(t+\tau))$. This function is readily a solution of the following system $\left(\mathcal{E}_{\tau}\right)$

$$
\dot{x}=y-F(x, \quad \dot{y}=-g(x)+\mu h(t+\tau),
$$

Take $h(t+\tau)$ as a sufficiently small perturbation of $h(t)$ as above. Therefore, according to the previous propositions, the solutions $\bar{\gamma}(\mathrm{t})$ and $\bar{\gamma}(\mathrm{t}+\tau)$ converge. Thus we obtain

$$
\|\bar{\gamma}(\mathrm{t}+\tau)-\bar{\gamma}(\mathrm{t})\|<\epsilon, \quad \mathrm{t}, \mathrm{t}+\tau \in \mathbb{R}-\mathrm{C}_{\epsilon} .
$$

Moreover we also have, for $t_{1}, t_{2} \in \mathbb{R}-C_{\epsilon}$,

$$
\left|\bar{\gamma}\left(t_{2}\right)-\bar{\gamma}\left(t_{1}\right)\right| \leq\left|t_{2}-t_{1}\right| \sup _{R}|\dot{\bar{\gamma}}|,
$$

which ensures the existence of $\delta$ such that

$$
\left|t_{1}-t_{2}\right|<\delta \Longrightarrow\left\|\bar{\gamma}\left(t_{1}\right)-\bar{\gamma}\left(t_{2}\right)\right\|<\epsilon, \quad t_{1}, t_{2} \in \mathbb{R}-C_{\epsilon},
$$

Therefore we conclude that the unique solution $\bar{\gamma}(\mathrm{t})$ is pseudo-almost periodic.

Moreover, from the previous lemma, all other solutions of the system that ultimately settle in $\mathrm{R}$ converge to this unique pseudo-almost periodic solution $\bar{\gamma}(\mathrm{t}) \in \mathrm{R}$. Therefore the system has a unique (isolated) almost periodic solution to which any other solution unwinds in the $\mathrm{C}^{1}$-bounded set R. It is a stable pseudo-almost limit cycle as defined above. Hence the claim.

\section{Pseudo-almost periodic Waves}

The importance of Liénard systems among nonlinear systems also comes from the fact that several systems can be transformed into Liénard systems and solved. [1, 19. We present next some partial differential equations solvable first by reducing them to some Liénard-type equations, then by applying the previous theorems.

\subsection{Hyperbolic pseudo-almost periodic Wave}

Consider systems described by the time-perturbed nonlinear hyperbolic equation

$$
u_{t t}=u_{x x}+f_{0}(u) u_{x}+g_{0}(u)+p(t)
$$


The search of special solutions of the form

$$
u(x, t)=y(x+\lambda t), \quad \lambda \in \mathbb{R}
$$

defining the wave with speed $v=|\lambda|$, yields the Liénard-type equation

$$
\left(1-\lambda^{2}\right) \ddot{y}+f_{0}(y) \dot{y}+g_{0}(y)=-p(t)
$$

Define $f(y)=\frac{f_{0}(y)}{1-\lambda^{2}}, g(y)=\frac{g_{0}(y)}{\left.1-\lambda^{2}\right)}$, and $h(t)=\frac{-p(t)}{1-\lambda^{2}}$. The functions $f_{0}$ and $g_{0}$ are continuously differentiable chosen together with the speed $v=|\lambda|$ of the waves $u(t, x)$ such that the function $f, g$, and $h$ satisfy the assumptions $L_{1}, \ldots, L_{4}$. Obviously assuming $p(t)$ pseudo-almost periodic implies $h(t)$ is pseudo-almost periodic. Therefore we conclude under these assumptions

Theorem 4.1. For a pseudo-almost periodic perturbation $\mathrm{p}(\mathrm{t})$, the nonlinear hyperbolic equation $(\mathcal{H})$ has a pseudo-almost periodic solitary wave $\mathrm{u}(\mathrm{x}, \mathrm{t})=\mathrm{y}(\mathrm{x}+\lambda \mathrm{t})$, where $\mathrm{y}(\mathrm{x})$ is the unique pseudo-almost limit cycle of the perturbed Liénard-type equation (4.2).

Proof. The proof is immediate and is adapted from theorem (3.6).

We next consider a parabolic partial differential equation describing a reaction-diffusion model.

\subsection{Parabolic pseudo-almost periodic Wave: a reaction-diffusion model}

Consider now the time-perturbed parabolic equation describing a reaction-diffusion model

$$
u_{t}=u_{x x}+f_{0}(u) u_{x}+g_{0}(u)+p(t) \quad(\mathcal{R D})
$$

Looking again for special solutions of the form (4.1) leads to the Liénard-type equation

$$
\ddot{y}+\left[f_{0}(y)-\lambda\right] \dot{y}+g_{0}(y)=0
$$

As in the previous case we set $f(y)=f_{0}(y)-\lambda, g(y)=g_{0}(y)$, and $h(t)=-p(t)$. The functions $f_{0}$ and $g_{0}$ are continuously differentiable and determined together with the speed $|\lambda|$ of the waves $u(t, x)$ such that the function $f, g$, and $h$ satisfy the assumptions $L_{1}, \ldots, L_{4}$. Again assuming $p(t)$ pseudo-almost periodic implies $h(t)$ is also pseudo-almost periodic. We therefore obtain the equivalent theorems of existence of pseudo-almost solitary waves to the reaction-diffusion equation as functions of the corresponding Liénard pseudo-almost limit cycles. That is,

Theorem 4.2. For a pseudo-almost periodic perturbation $\mathrm{p}(\mathrm{t})$, the nonlinear parabolic equation $(\mathcal{R} \mathcal{D})$ has a pseudo-almost periodic solitary wave $\mathrm{u}(\mathrm{x}, \mathrm{t})=\mathrm{y}(\mathrm{x}+\lambda \mathrm{t})$, where $\mathrm{y}(\mathrm{x})$ is the unique pseudoalmost limit cycle of the perturbed Liénard-type equation (4.3). 


\title{
5 Outlook and Open Problems
}

\author{
Arnold in [3] states
}

Une trajectoire fermée nondégénérée ne disparait pas par une petite déformation du système, mais se déforme légèrement. Donc le système des trajectoires est structurellement stable dans le voisinage de la trajectoire fermée générique

That is, periodic orbits do not just disappear under small perturbation, but they may be slightly deformed, due to the fact that the system of trajectories is structurally stable in the neighborhood of a periodic orbit. Many forced systems such as the Liénard ones are actually small perturbations of systems having periodic orbits (limit cycles) in their unperturbed form, and many results do imply the disappearance of these orbits upon perturbation. The appearing of pseudo-almost periodic solutions could result from the deformation/bifurcation of existing orbits. Therefore one must investigate the relation between the "new" pseudo-almost periodic solutions appearing upon perturbation and the periodic-type orbits of the unperturbed system, including the question in the following Open Problem 1.

\section{(1) Open Problem 1: Co-existence of limit cycles and strictly pseudo-almost limit cycles}

For parameterized systems, including the above Liénard systems, investigate conditions under which co-exist limit cycles and strictly almost or pseudo-almost limit cycles partitioning the phase space.

\section{(2) Open Problem 2: Isochronous pseudo-almost limit cycles}

Let $\gamma$ be a strictly pseudo-almost limit cycle of a flow $\phi$ on $\mathbb{R}^{n}$. A point $x_{1}$ in $\mathbb{R}^{n}$ has asymptotic phase with respect to $\gamma$ if there is a point $x_{0} \in \gamma$ such that $\lim _{\mathrm{t}} \longrightarrow \pm \infty \mid \phi_{\mathrm{t}}\left(x_{1}\right)-$ $\phi_{\mathrm{t}}\left(x_{0}\right) \mid=0$. We say that $x_{1}$ is in phase with $x_{0}$.

It is well known that a hyperbolic limit cycle has some neighborhood where every point has asymptotic phase with respect to the limit cycle, due to the existence of invariant foliation. [8] Similar question needs to be addressed as well in case of strictly pseudo-almost limit cycles.

Definition 5.1. A strictly pseudo-almost limit cycle is said to be isochronous if there is a neighborhood of $\gamma$ in which every point is in phase with a point on $\gamma$.

In the case of limit cycles, we have, for instance, the following examples. System

$$
\dot{\mathrm{r}}=-\frac{1}{3}(\mathrm{r}-1)^{4} e^{|\mathrm{r}-1|^{-3}}, \quad \dot{\theta}=2 \pi
$$

has a nonhyperbolic limit cycle at the unit cycle with period 1, attracting for $r>1$. The asymptotic phase of any point $\left(r_{0}, \theta_{0}\right)$ in its neighborhood is $\left(1, \theta_{0}\right)$. The limit cycle is therefore isochronous. For more details see [8].

It would be interesting to: 
(a) Perturb system (5.1), in particular in the angle variable, and study the conditions of appearance of strictly pseudo-almost limit cycles.

(b) Investigate the conditions of existence of isochronous strictly pseudo-almost limit cycles, in particular for the forced Liénard systems.

(c) Investigate the bifurcation of pseudo-almost limit cycles from an isochronous period annulus, as in [25]

(3) Open Problem 3: Pseudo-almost isochrons

As above, we further define:

Definition 5.2. Given $x_{0} \in \gamma$ where $\gamma$ is a strictly pseudo-almost limit cycle, a pseudoalmost isochron $\mathrm{I}\left(\mathrm{x}_{0}\right)$ based at $\mathrm{x}_{0}$ is the set of all point $\mathrm{x} \in \mathbb{R}^{\mathrm{n}}$ in phase with $\mathrm{x}_{0}$.

As in the case of limit cycles we conjecture the existence of pseudo-almost isochrons, and that they will foliate the neighborhood of pseudo-almost limit cycles. Their determination is definitely an interesting but difficult question of research. One line of attack might be similar to Guckenheimer and Winfree investigation of isochrons of limit cycles. [16, 26]

Received: October 2012. Revised: March 2013.

\section{References}

[1] Albarakati, W.A., Lloyd, N.G., J.M. Pearson, Transformation to Liénard form EJDE 2000(76), 1-11 (2000)

[2] Andronov, A.A. et al., Theory of Oscillators Dover, New York (1989)

[3] Arnold, V, Chapites supplémentaires de la Théorie des équations différentielles ordinaires Editions MIR, Moscou (1978)

[4] Bohr, H.A., Almost Periodic Functions Chelsea, New York (1951)

[5] Brauer, S.G. and Nohel, J.A., The qualitative theory of ordinary differential equations W.A. Benjamin New York (1968)

[6] Byrnes, C., Topological Methods for Nonlinear Oscillations Notices of the AMS, 57(9), 1080$1091(2010)$

[7] Cartwright M.L. and Littlewood, J.E., On non-linear differential equations of the second order II Annals of Maths 48(2), 472-494 (1947)

[8] Chicone, C. and Liu, W., Asymptotic Phase Revisited J.Diff.Equat 204, 227-246 (2004) 
[9] Christopher, C. and Li, C., Limit Cycles of Differential Equations Birkhauser Verlag, BaselBoston-Berlin (2007)

[10] Coddington, E.A. and Levinson, N, Theory of Ordinary Differential Equations Mc-Graw-Hill, NY (1953)

[11] Corduneanu, C., Almost Periodic Oscillations and Waves Springer (2009)

[12] Diagana, T., Pseudo Almost Periodic Functions in Banach Spaces Nova Publishers, Inc. New York (2007)

[13] Dumortier, F., Qualitative Theory of Planar Differential Systems Springer (2006)

[14] Ecalle, J. et al., Non-Accumulation des Cycles Limites I-II C.R.Acad.Sci. Paris. I(304), 375-431 (1987)

[15] Fink, A.M., Convergence and almost periodicity of solutions of forced Liénard equations SIAM J.Appl.Math. 26(1), 6-34 (1974)

[16] Guckenheimer, J. Isochrons and Phaseless sets J.Math.Biol. 1, 259-273 (1975)

[17] Hilbert, D., Mathematische Probleme The second International Congress of Mathematicians, Paris, 1900 Nachr.Ges.Wiss. Gottingen Math-Phys Kl 1900 253-297 (1900)

[18] Jordan, D.W. and Smith, P. Nonlinear Ordinary Differential Equations, Fourth Edition, Oxford University Press (2007)

[19] Lloyd, N.G., Liénard systems with several limit cycles Math.Proc.Camb.Phil.Soc. 102(565) (1987)

[20] N'Guérékata, G.M., Almost Automorphic Functions and Almost Periodic Functions in Abstract Spaces Kluwer Academic/Plenum Publishers, New York-London-Moscow (2001)

[21] Opial, Z., Sur les solutions périodiques et presque-périodiques de l'équations differentielle $x^{\prime \prime}+k f(x) x^{\prime}+g(x)=k p(t)$. Annales Polonici Mathematici, VII, 309-319 (1960)

[22] Poincaré, H., Mémoire sur les courbes définies par une équation differentielle J.Maths.Pures.Appli. 7, 375-422 (1881)

[23] Reuters, G.E.H., On certain non-linear differential equaions with almost periodic solutions Journal London Math.Soc. 26, 215-221 (1951)

[24] Toni, B., Almost and pseudo-almost limit cycles for some forced Liénard systems Nonlinear Analysis 71, 4718-4724 (2009)

[25] Toni, B., Upper Bounds of Limit Cycles from isochronous period annulus via birational linearization Discrete and Continuous Systems Supp(2005) 846-853 (2005) 
[26] Winfree, A.T., Patterns of Phase Compromise in Biological Cycles Journal of Mathematical Biology, 1, 73-95 (1974)

[27] Zhang, C., Almost Periodic type functions and Ergodicity Science Press (2003) 